\title{
NORM ESTIMATES FOR $k$-PLANE TRANSFORMS AND GEOMETRIC INEQUALITIES
}

\author{
B. RUBIN
}

\begin{abstract}
The article is devoted to remarkable interrelation between the norm estimates for $k$-plane transforms in weighted and unweighted $L^{p}$ spaces and geometric integral inequalities for crosssections of measurable sets in $\mathbb{R}^{n}$. We also consider more general $j$-plane to $k$-plane transforms on affine Grassmannians and their compact modifications. The article contains a series of new integral-geometric inequalities with sharp constants, explicit equalities, conjectures, and open problems.
\end{abstract}

\section{INTRODUCTION}

Let $\mathcal{A}_{n, k}$ be the affine Grassmannian bundle of all unoriented $k$ dimensional planes in $\mathbb{R}^{n}, 0<k<n$. We denote by $G_{n, k}$ the Grassmann manifold of $k$-dimensional linear subspaces of $\mathbb{R}^{n}$, i.e., $k$-planes passing through the origin. Each $k$-plane $\tau \in \mathcal{A}_{n, k}$ will be parameterized by the pair $(\xi, u)$, where $\xi \in G_{n, k}$ and $u \in \xi^{\perp}$, the orthogonal complement of $\xi$ in $\mathbb{R}^{n}$. The manifold $\mathcal{A}_{n, k}$ is endowed with the product measure $d \tau=d_{*} \xi d u$, where $d_{*} \xi$ is the $O(n)$-invariant probability measure on $G_{n, k}$ and $d u$ denotes the Euclidean volume element on $\xi^{\perp}$.

We will be dealing with standard spaces $L^{p}\left(\mathcal{A}_{n, k}\right)$ and the corresponding the weighted spaces

$$
L_{\nu}^{p}\left(\mathcal{A}_{n, k}\right)=\left\{f:\|f\|_{p, \nu} \equiv\left|\left\|\left.\tau\right|^{\nu} f(\tau)\right\|_{p}<\infty\right\}, \quad 1 \leq p \leq \infty,\right.
$$

where $\|\cdot\|_{p}$ is the usual norm in $L^{p}\left(\mathcal{A}_{n, k}\right)$ and $|\tau|$ stands for the Euclidean distance from the origin to $\tau$.

The $k$-plane Radon-John transform of a sufficiently good function $f$ on $\mathbb{R}^{n}$ is a function $R_{k} f$ on $\mathcal{A}_{n, k}$ defined by

$$
\left(R_{k} f\right)(\tau) \equiv\left(R_{k} f\right)(\xi, u)=\int_{x \in \tau} f(x) d_{\tau} x,
$$

2010 Mathematics Subject Classification. Primary 44A12; Secondary 52A40.

Key words and phrases. Radon transforms, Grassmann manifolds, geometric inequalities. 
where $d_{\tau} x$ is the Euclidean volume element on $\tau$. A more general $j$ plane to $k$-plane transform (or $(j, k)$-transform for short) takes a function $f$ on $\mathcal{A}_{n, j}$ to a function $R_{j, k} f$ on $\mathcal{A}_{n, k}, 0 \leq j<k<n$, by the formula

$$
\left(R_{j, k} f\right)(\tau) \equiv\left(R_{j, k} f\right)(\xi, u)=\int_{\zeta \subset \tau} f(\zeta) d_{\tau} \zeta .
$$

Here $d_{\tau} \zeta$ stands for the canonical measure on the affine Grassmann manifold of all $j$-dimensional planes in $\tau$. In the case $j=0,0$-planes are just points, so that $\mathcal{A}_{n, 0}=\mathbb{R}^{n}$ and $R_{0, k}=R_{k}$.

A background information about $k$-plane transforms can be found, e.g., in the books by Helgason [36], Markoe [44], and Gonzalez [28], containing many references on this subject; see also Keinert [37] and Rubin $[53,56]$. These publications are mainly focused on inversion and range characterization problems. More general $(j, k)$-transforms, which fall into the scope of the general Helgason's group-theoretic double fibration setting, were apparently first mentioned by Strichartz [66, p. 701]. The papers by Graev [30], Gonzalez and Kakehi [29], Rubin [54], Rubin and Wang [57, 58] deal with inversion formulas for these transforms. See also Strichartz [67] regarding $L^{2}$ harmonic analysis on Grassmannian bundles.

A tremendous activity related to $L^{p}-L^{q}$ norm estimates for Radon transforms was initiated by pioneering papers of Solmon [63, 64], Oberlin [47], Calderón [9], Oberlin and Stein [48], Strichartz [66], Christ [11], Drury [19, 20, 21, 22, 23]. Sharp constants and extremizers for Radonlike transforms were studied in the recent papers by Christ [12], Drouot [16, 17, 18], and Flock [24]: see also Baernstein II and Loss [2], Bennett, Bez and Gutirrez [3], Gressman [31, 32], Oberlin [49], Tao and Wright [68] for further developments and perspectives. Norm inequalities with sharp constants for $k$-plane transforms and $(j, k)$-transforms in weighted spaces (1.1) were obtained by Rubin [55].

Besides afore-mentioned purely analytic issues, our concern is related to the variety of publications in the area of integral geometry and probability dealing with volumes of cross-sections of geometrical objects. This direction of investigation amounts to the pioneering works by Steiner, Minkowski, Blaschke, and subsequent publications of many authors. Numerous related references can be found in the books by Burago and Zalgaller [6], Gardner [26], Schneider [61], Schneider and Weil [62]; see also the papers by Schneider [60], Grinberg [34], Lutwak [43], Gardner [27], Bianchi, Gardner and Gronchi [5], Chasapis, Giannopoulos and Liakopoulos [10], Dann, Kim and Yaskin [14], Dann, Paouris and Pivovarov [15], Dafnis and Paouris [13], to mention a few. 
In fact, the functional-analytic and geometric approaches are closely related because many results in analysis can be converted into similar statements in geometry, and vice versa. For example, an intimate connection of the Busemann intersection inequality [7] to the Oberlin-Stein $L^{p}-L^{q}$ estimate for the Radon transform [48] was noticed by Lutwak $[43$, p. $162,(3)]$; see subsection 4.3 for details. One of our aims in the present article is to extend Lutwak's remarkable observation to more general Radon-like transforms, derive new inequalities, and formulate some conjectures.

Plan of the Paper. In Section 2 we review basic facts related to norm inequalities for operators (1.2)-(1.3) and consider the corresponding weighted and unweighted inequalities for planar sections of measurable sets in $\mathbb{R}^{n}$. The main focus is made on sharp constants and their asymptotics as $n \rightarrow \infty$. Section 3 contains description of the transition from Radon transforms on affine Grassmannians to Funk type transforms on the unit sphere and compact Grassmannians. The results of this section are used in Section 4 to obtain new geometric inequalities for dual intrinsic volumes and central sections of star sets.

Main Results. The main new results of the paper are the general inequalities (2.25) and (2.30), Conjectures 2.4 and 4.2, Theorems 3.3 and 3.6. The paper contains many consequences of these statements which might be of independent interest.

Notation. We will be using the same notation as in (1.1)-(1.3); $V_{n}(\cdot)$ denotes the $n$-dimensional volume function; $\sigma_{n-1}=2 \pi^{n / 2} / \Gamma(n / 2)$ is the area of the unit sphere $S^{n-1}$ in $\mathbb{R}^{n} ; d \theta$ stands for the surface element of $S^{n-1} ; d_{*} \theta\left(=d \theta / \sigma_{n-1}\right)$ is the corresponding normalized surface element. The notation $d_{*}(\cdot)$ is also used for the probability measure on Grassmann manifolds. We write $B_{n}=\left\{x \in \mathbb{R}^{n}:|x| \leq 1\right\}$ for the unit ball in $\mathbb{R}^{n} ; b_{n}$ denotes the volume of $B_{n}$, so that

$$
b_{n}=\frac{\sigma_{n-1}}{n}=\frac{\pi^{n / 2}}{\Gamma(n / 2+1)} .
$$

A compact subset of $\mathbb{R}^{n}$ is called a body if it is the closure of its interior. As usual, for $1 \leq p \leq \infty$, the notation $p^{\prime}$ stands for the dual exponent, so that $1 / p+1 / p^{\prime}=1$. We say that an integral under consideration exists in the Lebesgue sense if it is finite when the corresponding integrand is replaced by its absolute value. 


\section{Norm Estimates of $k$-Plane Transforms and Affine Sections of Measurable Sets}

In this section we review basic facts related to the action of $k$-plane transforms (1.2) and $(j, k)$-transforms (1.3) in weighted and unweighted $L^{p}$ spaces and derive some integral-geometric inequalities for affine sections of measurable sets in $\mathbb{R}^{n}$. Our main concern is sharp constants in the corresponding inequalities.

2.1. Weighted Norm Estimates. The following statements were proved in our paper [55, Theorems 1.1 and 1.2].

Theorem 2.1. Let $1 \leq p \leq \infty, \nu=\mu-k / p^{\prime}, \mu>k-n / p$,

$$
\omega_{k, p, \mu}(n)=\pi^{k / 2 p^{\prime}}\left(\frac{\Gamma(n / 2)}{\Gamma((n-k) / 2)}\right)^{1 / p} \frac{\Gamma((\mu+n / p-k) / 2)}{\Gamma((\mu+n / p) / 2)} .
$$

Then $R_{k}$ is a linear bounded operator from $L_{\mu}^{p}\left(\mathbb{R}^{n}\right)$ to $L_{\nu}^{p}\left(\mathcal{A}_{n, k}\right)$ with the norm

$$
\left\|R_{k}\right\|=\omega_{k, p, \mu}(n) .
$$

Theorem 2.2. Let $1 \leq p \leq \infty, \nu=\mu-(k-j) / p^{\prime}, \mu>k-n / p-j / p^{\prime}$,

$$
\omega_{j, k, p, \mu}(n)=\pi^{(k-j) / 2 p^{\prime}}\left(\frac{\Gamma((n-j) / 2)}{\Gamma((n-k) / 2)}\right)^{1 / p} \frac{\Gamma\left(\left(\mu+n / p-k+j / p^{\prime}\right) / 2\right)}{\Gamma((\mu+n / p-j / p) / 2)} .
$$

Then $R_{j, k}$ is a linear bounded operator from $L_{\mu}^{p}\left(\mathcal{A}_{n, j}\right)$ to $L_{\nu}^{p}\left(\mathcal{A}_{n, k}\right)$ with the norm

$$
\left\|R_{j, k}\right\|=\omega_{j, k, p, \mu}(n) .
$$

Some comments are in order.

1. The assumptions for $\mu$ and $\nu$ in these theorems are best possible. Theorem 2.1 is formally contained in Theorem 2.2, in particular, $\omega_{k, p, \mu}(n)=\omega_{0, k, p, \mu}(n)$.

2. Theorems 2.1 and 2.2 were obtained in [55] as consequences of the corresponding norm inequalities for functions. For example, to prove (2.2), we established that

$$
\left\|R_{k} f\right\|_{L_{\nu}^{p}\left(\mathcal{A}_{n, k}\right)} \leq \omega_{k, p, \mu}(n)\|f\|_{L_{\mu}^{p}\left(\mathbb{R}^{n}\right)}
$$

for every $f \in L_{\mu}^{p}\left(\mathbb{R}^{n}\right)$ and

$$
\left\|R_{k} f_{0}\right\|_{L_{\nu}^{p}\left(\mathcal{A}_{n, k}\right)} \geq \omega_{k, p, \mu}(n)\left\|f_{0}\right\|_{L_{\mu}^{p}\left(\mathbb{R}^{n}\right)}
$$

for some nonnegative $f_{0} \in L_{\mu}^{p}\left(\mathbb{R}^{n}\right)$.

It means that (2.5) is sharp in the class of nonnegative functions in $L_{\mu}^{p}\left(\mathbb{R}^{n}\right)$, which is smaller than the entire space $L_{\mu}^{p}\left(\mathbb{R}^{n}\right)$. This remark will guarantee the sharpness of geometric inequalities in Subsection 4.2. 
3. In the case $p=1$, we have exact equalities

$$
\begin{aligned}
\int_{\mathcal{A}_{n, k}}\left(R_{k} f\right)(\tau)|\tau|^{\mu} d \tau & =\omega_{k, 1, \mu}(n) \int_{\mathbb{R}^{n}} f(x)|x|^{\mu} d x, \\
\int_{\mathcal{A}_{n, k}}\left(R_{j, k} f\right)(\tau)|\tau|^{\mu} d \tau & =\omega_{j, k, 1, \mu}(n) \int_{\mathcal{A}_{n, j}} f(\zeta)|\zeta|^{\mu} d \zeta,
\end{aligned}
$$

where $\mu>k-n$,

$$
\begin{gathered}
\omega_{k, 1, \mu}(n)=\frac{\Gamma(n / 2)}{\Gamma((n-k) / 2)} \frac{\Gamma((\mu+n-k) / 2)}{\Gamma((\mu+n) / 2)}, \\
\omega_{j, k, 1, \mu}(n)=\frac{\Gamma((n-j) / 2)}{\Gamma((n-k) / 2)} \frac{\Gamma((\mu+n-k) / 2)}{\Gamma((\mu+n-j) / 2)} .
\end{gathered}
$$

These formulas can be found in our papers [53, formula (2.17)] and [54, formula (2.20)] in different notation. It is assumed that either side of (2.7) and (2.8) exists in the Lebesgue sense.

4. We do not know if the exact equalities

$$
\begin{gathered}
\left\|R_{k} f\right\|_{L_{\nu}^{p}\left(\mathcal{A}_{n, k}\right)}=\omega_{k, p, \mu}(n)\|f\|_{L_{\mu}^{p}\left(\mathbb{R}^{n}\right)}, \\
\left\|R_{j, k} f\right\|_{L_{\nu}^{p}\left(\mathcal{A}_{n, k}\right)}=\omega_{j, k, p, \mu}(n)\|f\|_{L_{\mu}^{p}\left(\mathcal{A}_{n, j}\right)},
\end{gathered}
$$

are available for some $f$ when $p \neq 1$. It may be a challenging open problem.

5. One can easily find asymptotics of the norms (2.2) and (2.4) as $n \rightarrow \infty$. Indeed, the well-known property of gamma functions

$$
\Gamma(z+a) / \Gamma(z+b) \sim z^{a-b}, \quad z \rightarrow \infty,
$$

(see, e.g., [69]) yields

$$
\begin{gathered}
\omega_{j, k, p, \mu}(n)=n^{-(k-j) / 2 p^{\prime}}\left(\omega_{j, k, p}^{0}+o(1)\right), \quad n \rightarrow \infty . \\
\omega_{j, k, p}^{0}=(2 \pi)^{(k-j) / 2 p^{\prime}} p^{(k-j) / 2} .
\end{gathered}
$$

2.2. Unweighted $L^{p}-L^{q}$ Estimates. Let $0 \leq j<k<n$. It is known [54, Corollary 2.6] that for $f \in L^{p}\left(\mathcal{A}_{n, j}\right)$, the integral $R_{j, k} f$ is finite provided $1 \leq p<(n-j) /(k-j)$ and this bound is sharp. By (2.8) with $\mu=0, R_{j, k}$ acts as a linear bounded operator from $L^{1}\left(\mathcal{A}_{n, j}\right)$ to $L^{1}\left(\mathcal{A}_{n, k}\right)$ with the operator norm 1 . Further, by the scaling argument (cf. [65, p. 118]), if the estimate

$$
\left\|R_{j, k} f\right\|_{L^{q}\left(\mathcal{A}_{n, k}\right)} \leq c\|f\|_{L^{p}\left(\mathcal{A}_{n, j}\right)}
$$


holds for every $f \in L^{p}\left(\mathcal{A}_{n, j}\right)$ with a constant $c$ independent of $f$, then, necessarily,

$$
1 / q=\frac{n-j}{n-k}(1 / p)-\frac{k-j}{n-k}
$$

It means that the set of all pairs $(1 / p, 1 / q)$ which are admissible in (2.15), is a subset of the half-open segment $(P, Q]$ in $[0,1] \times[0,1]$ connecting the points $P=((k-j) /(n-j), 0)$ and $\mathrm{Q}=(1,1)$. To achieve the desired boundedness result, the segment $(P, Q]$ must be replaced by the smaller closed segment $[\tilde{P}, Q]$ with $\tilde{P}=((k+1) /(n+1), 1 /(n+1))$, and we have the following theorem.

Theorem 2.3. Let $0<k<n$,

$$
p=\frac{n+1}{k+1}, \quad q=n+1, \quad \Omega_{k}(n)=\left(2^{k-n} \frac{\sigma_{k}^{n}}{\sigma_{n}^{k}}\right)^{1 /(n+1)} .
$$

Then

$$
\left\|R_{k} f\right\|_{L^{q}\left(\mathcal{A}_{n, k}\right)} \leq \Omega_{k}(n)\|f\|_{L^{p}\left(\mathbb{R}^{n}\right)} .
$$

The equality sign in (2.17) holds if and only if

$$
f(x)=c\left(1+|M x|^{2}\right)^{-(k+1) / 2},
$$

where $c=$ const and $M$ is an invertible affine map.

This remarkable theorem combines results of several authors. The estimate (2.17) with unspecified numeric constant is due to Oberlin and Stein [48], Christ [11], and Drury [19, 20, 22]. The question of optimal constant and extremizers in (2.17) was first considered by Baernstein and Loss [2] who made several important conjectures regarding (2.17) and related problems. A sharp constant $\Omega_{k}(n)$ was found by Drouot [16]. The equality case in (2.17) for $k=n-1$ was studied by Christ [12]. The choice of the extremizer (2.18) for all $0<k<n$ was justified by Drouot $[16,17]$ and Flock [24].

The estimate (2.17) extends to all $1 \leq p \leq(n+1) /(k+1)$ by interpolation and the bound $(n+1) /(k+1)$ is sharp.

To the best of our knowledge, a complete analogue of Theorem 2.3 for $(j, k)$-transforms is unknown, and we formulate the following conjecture.

Conjecture 2.4. Let $0 \leq j<k<n$,

$$
p=\frac{n+1}{k+1}, \quad q=\frac{n+1}{j+1}, \quad \Omega_{j, k}(n)=\left(\sigma_{j}^{k-n} \sigma_{k}^{n-j} \sigma_{n}^{j-k}\right)^{1 /(n+1)} .
$$

Then

$$
\left\|R_{j, k} f\right\|_{L^{q}\left(\mathcal{A}_{n, k}\right)} \leq \Omega_{j, k}(n)\|f\|_{L^{p}\left(\mathcal{A}_{n, j}\right)}
$$


where

$$
\Omega_{j, k}(n)=\left(\sigma_{k}^{n-j} \sigma_{j}^{k-n} \sigma_{n}^{j-k}\right)^{1 /(n+1)}
$$

is the norm of the operator $R_{j, k}$. The equality sign in (2.19) holds if and only if

$$
f(\zeta)=c\left(1+|M \zeta|^{2}\right)^{-(k+1) / 2}, \quad \zeta \in \mathcal{A}_{n, j},
$$

where $c=$ const and $M$ is an invertible affine map.

Clearly, for $j=0$ we have $\Omega_{0, k}(n)=\Omega_{k}(n)$, as in $(2.17)$.

The boundedness of $R_{j, k}$ from $L^{(n+1) /(k+1)}\left(\mathcal{A}_{n, j}\right)$ to $L^{(n+1) /(j+1)}\left(\mathcal{A}_{n, k}\right)$ was stated by Drury in [23, Theorem 2], [22, formula (12)]. Conjecture 2.4 is partly motivated by the following lemma, according to which

$$
\left\|R_{j, k}\right\| \geq \Omega_{j, k}(n) .
$$

Lemma 2.5. If $f_{0}(\zeta)=\left(1+|\zeta|^{2}\right)^{-(k+1) / 2}, \zeta \in \mathcal{A}_{n, j}$, then

$$
\left\|R_{j, k} f_{0}\right\|_{L^{q}\left(\mathcal{A}_{n, k}\right)}=\Omega_{j, k}(n)\left\|f_{0}\right\|_{L^{p}\left(\mathcal{A}_{n, j}\right)},
$$

where all parameters have the same meaning as in Conjecture 2.4.

Proof. By [54, formula $(2.12)],\left(R_{j, k} f_{0}\right)(\tau)=\lambda\left(1+|\tau|^{2}\right)^{-(j+1) / 2}$, where

$$
\lambda=\frac{\pi^{(k-j) / 2} \Gamma((j+1) / 2)}{\Gamma((k+1) / 2)}=\frac{\sigma_{k}}{\sigma_{j}} .
$$

Note also that

$$
\left\|f_{0}\right\|_{L^{p}\left(\mathcal{A}_{n, j}\right)}^{p}=\int_{\mathcal{A}_{n, j}}\left(1+|\zeta|^{2}\right)^{-(n+1) / 2} d \zeta=\sigma_{n-j-1} \int_{0}^{\infty} \frac{t^{n-j-1} d t}{\left(1+t^{2}\right)^{(n+1) / 2}} d t=\frac{\sigma_{n}}{\sigma_{j}}
$$

(use, e.g., [33, formula $3.196(2)]$ ). Similarly,

$$
\left\|R_{j, k} f_{0}\right\|_{L^{q}\left(\mathcal{A}_{n, k}\right)}^{q}=\lambda^{q} \int_{\mathcal{A}_{n, k}}\left(1+|\tau|^{2}\right)^{-(n+1) / 2} d \zeta=\lambda^{q} \frac{\sigma_{n}}{\sigma_{k}}=\left(\frac{\sigma_{k}}{\sigma_{j}}\right)^{q} \frac{\sigma_{n}}{\sigma_{k}} .
$$

This gives (2.23).

Remark 2.6. The following intriguing inequality for the $k$-plane transform due to Dann, Paouris and Pivovarov [15, Theorem 1.3] might be a nice addition to this subsection.

Theorem 2.7. If $f$ is a nonnegative bounded integrable function on $\mathbb{R}^{n}, 0<k<n$, then

$$
\int_{\mathcal{A}_{n, k}}\left[\left(R_{k} f\right)(\tau)\right]^{n+1} \frac{d \tau}{\left\|\left.f\right|_{\tau}\right\|_{\infty}^{n-k}} \leq \frac{b_{k}^{n+1} b_{n(k+1)}}{b_{n}^{k+1} b_{k(n+1)}}\|f\|_{1}^{k+1},
$$

where $\left.f\right|_{\tau}$ is the restriction of $f$ onto $\tau$. 
2.3. Volume Estimates for Cross-Sections of Sets in $\mathbb{R}^{n}$. Inequalities for Radon transforms in the previous subsections give rise to a series of estimates for cross-sections of measurable subsets of $\mathbb{R}^{n}$. The only restriction on these subsets is the finiteness of the right-hand side in the corresponding estimates. The estimates contain several parameters that can be chosen depending on our need.

2.3.1. Weighted Estimates. Given a measurable set $S$ in $\mathbb{R}^{n}$, let $\mathbf{1}_{S}(x)$ be the indicator function of $S$, that is, $\mathbf{1}_{S}(x) \equiv 1$ if $x \in S$ and $\mathbf{1}_{S}(x) \equiv 0$ if $x \notin S$. Then, for $\tau \in \mathcal{A}_{n, k}$, we have $V_{k}(S \cap \tau)=\left(R_{k} \mathbf{1}_{S}\right)(\tau)$, and Theorem 2.1 yields the following statement.

Proposition 2.8. Let

$$
0<k<n, \quad 1 \leq p \leq \infty, \quad \nu=\mu-k / p^{\prime}, \quad \mu>k-n / p .
$$

Then

$$
\int_{\mathcal{A}_{n, k}}\left[V_{k}(S \cap \tau)\right]^{p}|\tau|^{\nu p} d \tau \leq \omega_{k, p, \mu}^{p}(n) \int_{S}|x|^{\mu p} d x,
$$

where $\omega_{k, p, \mu}(n)$ is defined by (2.1), so that

$$
\omega_{k, p, \mu}(n)=n^{-k / 2 p^{\prime}}\left((2 \pi)^{k / 2 p^{\prime}} p^{k / 2}+o(1)\right), \quad n \rightarrow \infty .
$$

Note that the principal term of the asymptotics of $\omega_{k, p, \mu}(n)$ is independent of $\mu$. The case $\mu=0$ gives the following corollary.

Corollary 2.9. If $1 \leq p<n / k$, then

$$
\begin{gathered}
\int_{\mathcal{A}_{n, k}}\left[V_{k}(S \cap \tau)\right]^{p}|\tau|^{-k(p-1)} d \tau \leq \omega_{k, p, 0}^{p}(n) V_{n}(S), \\
\omega_{k, p, 0}(n)=\pi^{k / 2 p^{\prime}}\left(\frac{\Gamma(n / 2)}{\Gamma((n-k) / 2)}\right)^{1 / p} \frac{\Gamma((n / p-k) / 2)}{\Gamma(n / 2 p)} .
\end{gathered}
$$

Remark 2.10. If $p=1, \mu>k-n$, then (2.7) yields an explicit equality

$$
\int_{\mathcal{A}_{n, k}} V_{k}(S \cap \tau)|\tau|^{\mu} d \tau=\omega_{k, 1, \mu}(n) \int_{S}|x|^{\mu} d x,
$$

where $\omega_{k, 1, \mu}(n)$ is a constant (2.9). In particular, for $\mu=0$,

$$
\int_{\mathcal{A}_{n, k}} V_{k}(S \cap \tau) d \tau=V_{n}(S)
$$

which is a well-known consequence of Fubini's theorem. 
More generally, assuming $\zeta \in \mathcal{A}_{n, j}, \tau \in \mathcal{A}_{n, k}, 0 \leq j<k<n$, and setting $f(\zeta)=\left(R_{j} \mathbf{1}_{S}\right)(\zeta)=V_{j}(S \cap \zeta)$, we obtain

$$
\left(R_{j, k} f\right)(\tau)=\left(R_{j, k}\left[R_{j} \mathbf{1}_{S}\right]\right)(\tau)=\left(R_{k} \mathbf{1}_{S}\right)(\tau)=V_{k}(S \cap \tau) .
$$

Now, Theorem 2.2 gives the following inequality for mean volumes of cross-sections of different dimensions:

$$
\int_{\mathcal{A}_{n, k}}\left[V_{k}(S \cap \tau)\right]^{p}|\tau|^{\nu p} d \tau \leq \omega_{j, k, p, \mu}^{p}(n) \int_{\mathcal{A}_{n, j}}\left[V_{j}(S \cap \zeta)\right]^{\mu p} d \zeta .
$$

Here $\mu, \nu, p$ and $\omega_{j, k, p, \mu}(n)$ have the same meaning as in Theorem 2.2. 2.3.2. Unweighted Estimates. The inequality (2.17) yields

$$
\int_{\mathcal{A}_{n, k}}\left[V_{k}(S \cap \tau)\right]^{n+1} d \tau \leq 2^{k-n} \frac{\sigma_{k}^{n}}{\sigma_{n}^{k}}\left[V_{n}(S)\right]^{k+1} .
$$

A more general inequality follows from (2.19) and has the form

$$
\begin{aligned}
& \left(\int_{\mathcal{A}_{n, k}}\left[V_{k}(S \cap \tau)\right]^{(n+1) /(j+1)} d \tau\right)^{(j+1) /(n+1)} \\
& \leq \Omega_{j, k}(n)\left(\int_{\mathcal{A}_{n, j}}\left[V_{j}(S \cap \zeta)\right]^{(n+1) /(k+1)} d \zeta\right)^{(k+1) /(n+1)}
\end{aligned}
$$

provided that Conjecture 2.4 is true.

Remark 2.11. Apparently the weighted inequalities (2.25) and (2.27) are not sharp because the subclass of indicator functions is much smaller than that in Theorems 2.1 and 2.2. Regarding unweighted case, the following sharp result is due to Gardner [27, Theorem 7.8]. For the sake of convenience, we formulate it in our notation.

Theorem 2.12. Let $S$ be a bounded Borel set in $\mathbb{R}^{n}, 1 \leq k \leq n$. Then

$$
\int_{\mathcal{A}_{n, k}}\left[V_{k}(S \cap \tau)\right]^{n+1} d \tau \leq \frac{b_{k}^{n+1} b_{n(k+1)}}{b_{n}^{k+1} b_{k(n+1)}}\left[V_{n}(S)\right]^{k+1}
$$

with equality when $k>1$ if and only if $S$ is an $n$-dimensional ellipsoid, modulo a set of measure zero, and when $k=1$ if and only if $S$ is a convex body, modulo a set of measure zero.

The estimate (2.33) is sharper than (2.31) and agrees with (2.24). A more general inequality

$$
\int_{\mathcal{A}_{n, k}}\left[V_{k}(S \cap \tau)\right]^{m+1} d \tau \leq \frac{b_{k}^{m+1} b_{n+k m}}{b_{n}^{(n+k m) / n} b_{k+k m}}\left[V_{n}(S)\right]^{1+k m / n},
$$


where $S$ is a convex body and $m \in\{1, \ldots, n\}$ is due to Schneider [60]. Note that (2.33) and (2.34) assume the set $S$ to be bounded, while (2.31) holds for arbitrary (not necessarily bounded) measurable set of finite measure.

\section{Transition from $\mathbb{R}^{n}$ And Affine Grassmannians to the Sphere And COMPACT Grassmannians}

Theorems of the previous section can be converted into the similar statements for the Funk type transforms on the sphere and Grassmann manifolds by making use of the stereographic projection. A remarkable interrelation between diverse integral operators on $\mathbb{R}^{n}$ and $S^{n}$ is known for many years and the corresponding transition formulas can be found in numerous publications; see, e.g., Mikhlin [45, pp. 35-36], Berenstein, Casadio Tarabusi and Kurusa [4], Drury [23], Rubin [56, Section 5.2], to mention a few. Below we recall the reasoning from our paper [54] which has proved to be especially helpful in the general context of Grassmannians.

We consider the Euclidean space $\mathbb{R}^{n}=\mathbb{R} e_{1} \oplus \ldots \oplus \mathbb{R} e_{n}$ as a coordinate hyperplane in $\mathbb{R}^{n+1}=\mathbb{R} e_{1} \oplus \ldots \oplus \mathbb{R} e_{n+1}$. Given a linear subspace $V$ of $\mathbb{R}^{n+1}$ and a positive integer $k<\operatorname{dim} V$, we denote by $G_{k}(V)$ the Grassmann manifold of all $k$-dimensional linear subspaces of $V$. In particular, $G_{k}\left(\mathbb{R}^{n}\right)=G_{n, k}, G_{k+1}\left(\mathbb{R}^{n+1}\right)=G_{n+1, k+1}$. To each affine $k$ plane $\tau$ in $\mathbb{R}^{n}$ we associate a $(k+1)$-dimensional linear subspace $\tau_{0}$ in $\mathbb{R}^{n+1}$ containing the "lifted" plane $\tau+e_{n+1}$. This leads to a map

$$
\mathcal{A}_{n, k} \ni \tau=\xi+u \stackrel{\gamma_{k}}{\longrightarrow} \tau_{0}=\gamma_{k}(\tau)=\xi \oplus \mathbb{R} u_{0} \in G_{n+1, k+1},
$$

where

$$
u_{0}=\frac{u+e_{n+1}}{\left|u+e_{n+1}\right|}=\frac{u+e_{n+1}}{\sqrt{1+|u|^{2}}} \in S^{n} .
$$

If $\theta=d\left(\tau_{0}\right)$ is the geodesic distance (on $S^{n}$ ) between the north pole $e_{n+1}$ and the $k$-dimensional totally geodesic submanifold $S^{n} \cap \tau_{0}$, then

$$
|\tau|=|u|=\tan \theta \text {. }
$$

In a similar way, we define a map $\gamma_{j}$ by

$$
\begin{array}{r}
\mathcal{A}_{n, j} \ni \zeta=\eta+v \stackrel{\gamma_{j}}{\longrightarrow} \zeta_{0}=\gamma_{j}(\zeta)=\eta \oplus \mathbb{R} v_{0} \in G_{n+1, j+1}, \\
v_{0}=\frac{v+e_{n+1}}{\sqrt{1+|v|^{2}}} \in S^{n}, \quad|\zeta|=|v|=\tan \omega, \quad \omega=d\left(\zeta_{0}\right),
\end{array}
$$

and denote

$$
\begin{aligned}
& \left(\Lambda_{j} f\right)\left(\zeta_{0}\right)=f\left(\gamma_{j}^{-1}\left(\zeta_{0}\right)\right), \quad f \equiv f(\zeta), \quad \zeta \in \mathcal{A}_{n, j}, \\
& \left(\Lambda_{k} \varphi\right)\left(\tau_{0}\right)=\varphi\left(\gamma_{k}^{-1}\left(\tau_{0}\right)\right), \quad \varphi \equiv \varphi(\tau), \quad \tau \in \mathcal{A}_{n, k} .
\end{aligned}
$$


The maps $\gamma_{j}$ and $\gamma_{k}$ are one-to-one up to the corresponding subsets of measure zero.

For $0 \leq j<k<n$, consider the Funk type transform

$$
\left(F_{j+1, k+1} g\right)\left(\tau_{0}\right)=\int_{G_{j+1}\left(\tau_{0}\right)} g\left(\zeta_{0}\right) d_{\tau_{0}} \zeta_{0}, \quad \tau_{0} \in G_{n+1, k+1},
$$

that integrates $g\left(\zeta_{0}\right)$ over the set of all $(j+1)$-dimensional linear subspaces of $\tau_{0}$ against the canonical probability measure on $G_{j+1}\left(\tau_{0}\right)$. If $j=0,(3.5)$ is the classical Funk transform of even functions on $S^{n}$ $[36,56]$.

The following statement is a reformulation of Lemmas 3.2 and 3.4 from [54] adapted to our notation.

Lemma 3.1. Let $0 \leq j<k<n$,

$$
a=\frac{\sigma_{k}}{\sigma_{j}}, \quad \rho_{1}(\zeta)=\left(1+|\zeta|^{2}\right)^{-(k+1) / 2}, \quad \rho_{2}(\tau)=\left(1+|\tau|^{2}\right)^{-(j+1) / 2} .
$$

Then

$$
\begin{gathered}
R_{j, k} f=a \rho_{2} \Lambda_{k}^{-1} F_{j+1, k+1} \Lambda_{j} \rho_{1}^{-1} f, \quad f: \mathcal{A}_{n, j} \rightarrow \mathbb{C} \\
F_{j+1, k+1} g=a^{-1} \Lambda_{k} \rho_{2}^{-1} R_{j, k} \rho_{1} \Lambda_{j}^{-1} g, \quad g: G_{n+1, j+1} \rightarrow \mathbb{C} \\
\int_{\mathcal{A}_{n, k}} \varphi(\tau) d \tau=\frac{\sigma_{n}}{\sigma_{k}} \int_{G_{n+1, k+1}} \frac{\left(\Lambda_{k} \varphi\right)\left(\tau_{0}\right)}{\left(\cos d\left(\tau_{0}\right)\right)^{n+1}} d_{*} \tau_{0} \\
\int_{G_{n+1, k+1}} g\left(\tau_{0}\right) d_{*} \tau_{0}=\frac{\sigma_{k}}{\sigma_{n}} \int_{\mathcal{A}_{n, k}} \frac{\left(\Lambda_{k}^{-1} g\right)(\tau)}{\left(1+|\tau|^{2}\right)^{(n+1) / 2}} d_{\tau} .
\end{gathered}
$$

3.1. Unweighted Estimates. Lemma 3.1 implies the following statement.

Corollary 3.2. Let

$$
p=\frac{n+1}{k+1}, \quad q=\frac{n+1}{j+1}, \quad b=\left(\frac{\sigma_{j}}{\sigma_{n}}\right)^{1 / p}, \quad c=\left(\frac{\sigma_{k}}{\sigma_{n}}\right)^{1 / q} .
$$

Then

$$
\begin{aligned}
\left\|\Lambda_{j} \rho_{1}^{-1} f\right\|_{L^{p}\left(G_{n+1, j+1}\right)} & =b\|f\|_{L^{p}\left(\mathcal{A}_{n, j}\right)}, \\
\left\|\Lambda_{k} \rho_{2}^{-1} R_{j, k} f\right\|_{L^{q}\left(G_{n+1, k+1}\right)} & =c\left\|R_{j, k} f\right\|_{L^{q}\left(\mathcal{A}_{n, k}\right)} .
\end{aligned}
$$


Proof. Denote by $I$ and $J$ the left-hand sides of (3.12) and (3.13), respectively. By (3.10) (with $k$ replaced by $j$ ),

$$
I^{p}=\frac{\sigma_{j}}{\sigma_{n}} \int_{\mathcal{A}_{n, j}} \frac{\Lambda_{j}^{-1}\left[\left(\Lambda_{j} \rho_{1}^{-1} f\right)^{p}\right](\zeta)}{\left(1+|\zeta|^{2}\right)^{(n+1) / 2}} d \zeta=\frac{\sigma_{j}}{\sigma_{n}}\|f\|_{L^{p}\left(\mathcal{A}_{n, j}\right)}^{p} .
$$

Similarly,

$$
J^{q}=\frac{\sigma_{k}}{\sigma_{n}} \int_{\mathcal{A}_{n, k}} \frac{\Lambda_{k}^{-1}\left[\left(\Lambda_{k} \rho_{2}^{-1} R_{j, k} f\right)^{q}\right](\tau)}{\left(1+|\tau|^{2}\right)^{(n+1) / 2}} d \tau=\frac{\sigma_{k}}{\sigma_{n}}\left\|R_{j, k} f\right\|_{L^{q}\left(\mathcal{A}_{n, k}\right)}^{q} .
$$

Theorem 3.3. Let $0 \leq j<k<n$,

$$
p=\frac{n+1}{k+1}, \quad q=\frac{n+1}{j+1}, \quad \Omega_{j, k}(n)=\left(\sigma_{k}^{n-j} \sigma_{j}^{k-n} \sigma_{n}^{j-k}\right)^{1 /(n+1)} .
$$

(i) If $F_{j+1, k+1}$ is bounded from $L^{p}\left(G_{n+1, j+1}\right)$ to $L^{q}\left(G_{n+1, k+1}\right)$, then $R_{j, k}$ is bounded from $L^{p}\left(\mathcal{A}_{n, j}\right)$ to $L^{q}\left(\mathcal{A}_{n, k}\right)$ and

$$
\Omega_{j, k}(n) \leq\left\|R_{j, k}\right\| \leq \Omega_{j, k}(n)\left\|F_{j+1, k+1}\right\| .
$$

(ii) Conversely, if $R_{j, k}$ is bounded from $L^{p}\left(\mathcal{A}_{n, j}\right)$ to $L^{q}\left(\mathcal{A}_{n, k}\right)$, then $F_{j+1, k+1}$ is bounded from $L^{p}\left(G_{n+1, j+1}\right)$ to $L^{q}\left(G_{n+1, k+1}\right)$ and

$$
1 \leq\left\|F_{j+1, k+1}\right\| \leq \Omega_{j, k}^{-1}(n)\left\|R_{j, k}\right\| .
$$

Proof. (i) Using successively (3.13), (3.7) and (3.12), we obtain

$$
\begin{aligned}
\left\|R_{j, k} f\right\|_{q} & =\frac{1}{c}\left\|\Lambda_{k} \rho_{2}^{-1} R_{j, k} f\right\|_{q}=\frac{a}{c}\left\|F_{j+1, k+1} \Lambda_{j} \rho_{1}^{-1} f\right\|_{q} \\
& \leq \frac{a}{c}\left\|F_{j+1, k+1}\right\|\left\|\Lambda_{j} \rho_{1}^{-1} f\right\|_{p}=\frac{a b}{c}\left\|F_{j+1, k+1}\right\|\|f\|_{p} .
\end{aligned}
$$

Hence, $\left\|R_{j, k}\right\| \leq(a b / c)\left\|F_{j+1, k+1}\right\|=\Omega_{j, k}(n)\left\|F_{j+1, k+1}\right\|$. The left inequality in (3.14) mimics (2.22).

(ii) The successive use of (3.8), (3.13) and (3.12) yields

$$
\begin{aligned}
\left\|F_{j+1, k+1} g\right\|_{q} & =\frac{1}{a}\left\|\Lambda_{k} \rho_{2}^{-1} R_{j, k} \rho_{1} \Lambda_{j}^{-1} g\right\|_{q}=\frac{c}{a}\left\|R_{j, k} \rho_{1} \Lambda_{j}^{-1} g\right\|_{q} \\
& \leq \frac{c}{a}\left\|R_{j, k}\right\|\left\|\rho_{1} \Lambda_{j}^{-1} g\right\|_{p}=\frac{c}{a b}\left\|R_{j, k}\right\|\left\|\Lambda_{j} \rho_{1}^{-1} \rho_{1} \Lambda_{j}^{-1} g\right\|_{p} \\
& =\frac{c}{a b}\left\|R_{j, k}\right\|\|g\|_{p}=\Omega_{j, k}^{-1}(n)\left\|R_{j, k}\right\|\|g\|_{p} .
\end{aligned}
$$

Hence $\left\|F_{j+1, k+1}\right\| \leq \Omega_{j, k}^{-1}(n)\left\|R_{j, k}\right\|$. The left inequality in (3.15) follows from the obvious equality $\left\|F_{j+1, k+1} \mathbf{1}\right\|_{q}=\|\mathbf{1}\|_{p}=1$.

The inequalities (3.14) and (3.15) imply the following statement. 
Corollary 3.4. Suppose that Conjecture 2.4 is true. Then, for all $0 \leq j<k<n$,

$$
\left\|R_{j, k}\right\|=\Omega_{j, k}(n)\left\|F_{j+1, k+1}\right\|
$$

in the corresponding $L^{p}-L^{q}$ setting, as in Theorem 3.3. In particular, if $\left\|F_{j+1, k+1}\right\|=1$, then $\left\|R_{j, k}\right\|=\Omega_{j, k}(n)$.

In view of forthcoming applications, for the sake of convenience we replace $n$ by $n-1, k$ by $k-1$, and $j$ by $j-1$. Then for all $0 \leq j<k<n$, Corollary 3.4 implies

$$
\left(\int_{G_{n, k}}\left|\left(F_{j, k} \varphi\right)\left(\tau_{0}\right)\right|^{n / j} d_{*} \tau_{0}\right)^{j / n} \leq\left(\int_{G_{n, j}}\left|\varphi\left(\zeta_{0}\right)\right|^{n / k} d_{*} \zeta_{0}\right)^{k / n}
$$

If Conjecture 2.4 is true, this inequality is sharp. A similar inequality without sharp constant was outlined by Drury [22, formula (10)], [23, Theorem 1].

Remark 3.5. The case $j=0$ deserves particular mentioning. By Theorem $2.3,\left\|R_{k}\right\|=\Omega_{k}(n)$. Hence (3.16) yields

$$
\left\|F_{1, k}\right\|_{L^{p}\left(G_{n, 1}\right) \rightarrow L^{q}\left(G_{n, k}\right)}=1, \quad p=n / k, \quad q=n, \quad 0<k<n .
$$

Identifying functions on $G_{n, 1}$ with even functions on $S^{n-1}$ and noting that

$$
\left(F_{1, k} \varphi\right)\left(\tau_{0}\right) \equiv\left(F_{k} \varphi\right)\left(\tau_{0}\right)=\int_{S^{n-1} \cap \tau_{0}} \varphi(\theta) d_{\tau_{0}} \theta
$$

is the Funk type transform that integrates $\varphi$ over $(k-1)$-dimensional geodesics $S^{n-1} \cap \tau_{0}$ with respect to the corresponding probability measure [36, p. 133], we obtain a sharp inequality

$$
\left(\int_{G_{n, k}}\left|\left(F_{k} \varphi\right)\left(\tau_{0}\right)\right|^{n} d_{*} \tau_{0}\right)^{1 / n} \leq\left(\int_{S^{n-1}}|\varphi(\theta)|^{n / k} d_{*} \theta\right)^{k / n} .
$$

Because odd functions are annihilated by $F_{k}$, the assumption of evenness of $\varphi$ can be dropped. Indeed, setting $\varphi=\varphi_{+}+\varphi_{-}$, where 


$$
\begin{aligned}
\varphi_{ \pm}(\theta) & =[\varphi(\theta) \pm \varphi(-\theta)] / 2, \text { we have } \\
& \left(\int_{G_{n, k}}\left|\left(F_{k} \varphi\right)\left(\tau_{0}\right)\right|^{n} d_{*} \tau_{0}\right)^{1 / n}=\left(\int_{G_{n, k}}\left|\left(F_{k} \varphi_{+}\right)\left(\tau_{0}\right)\right|^{n} d_{*} \tau_{0}\right)^{1 / n} \\
\leq & \left(\int_{S^{n-1}}\left|\varphi_{+}(\theta)\right|^{n / k} d_{*} \theta\right)^{k / n}=\frac{1}{2}\left(\int_{S^{n-1}}|[\varphi(\theta)+\varphi(-\theta)]|^{n / k} d_{*} \theta\right)^{k / n} \\
\leq & \frac{1}{2}\left[\left(\int_{S^{n-1}}|\varphi(\theta)|^{n / k} d_{*} \theta\right)^{k / n}+\left(\int_{S^{n-1}}|\varphi(-\theta)|^{n / k} d_{*} \theta\right)^{k / n}\right] \\
& =\left(\int_{S^{n-1}}|\varphi(\theta)|^{n / k} d_{*} \theta\right)^{k / n} .
\end{aligned}
$$

The inequality (3.19) provides additional information to the corresponding results of Christ [11, Theorem 2.1 (B)] and Drury [22, Theorem 1], where the sharp constant is not specified. An alternative derivation of (3.19) can be found in Drouot [18, Theorem 2].

3.2. Weighted Estimates. We combine Lemma 3.1 with Theorem 2.2 and replace $n+1$ by $n, k+1$ by $k$, and $j+1$ by $j$, respectively. This gives the following statement.

Theorem 3.6. Let $0 \leq j<k<n, \zeta_{0} \in G_{n, j}, \tau_{0} \in G_{n, k}$,

$$
\begin{aligned}
& \alpha\left(\tau_{0}\right)=\left(\sin d\left(\tau_{0}\right)\right)^{\nu p}\left(\cos d\left(\tau_{0}\right)\right)^{(j-\nu) p-n}, \\
& \beta\left(\zeta_{0}\right)=\left(\sin d\left(\zeta_{0}\right)\right)^{\mu p}\left(\cos d\left(\zeta_{0}\right)\right)^{(k-\mu) p-n} .
\end{aligned}
$$

Suppose that

$$
1 \leq p \leq \infty, \quad \nu=\mu-(k-j) / p^{\prime}, \quad \mu>k-n / p-j / p^{\prime} .
$$

Then

$$
\left(\int_{G_{n, k}}\left|\left(F_{j, k} \varphi\right)\left(\tau_{0}\right)\right|^{p} \alpha\left(\tau_{0}\right) d_{*} \tau_{0}\right)^{1 / p} \leq c\left(\int_{G_{n, j}}\left|\varphi\left(\zeta_{0}\right)\right|^{p} \beta\left(\zeta_{0}\right) d_{*} \zeta_{0}\right)^{1 / p}
$$

where $c$ is a sharp constant having the form

$$
c=\left(\frac{\Gamma(k / 2)}{\Gamma(j / 2)}\right)^{1 / p^{\prime}}\left(\frac{\Gamma((n-j) / 2)}{\Gamma((n-k) / 2)}\right)^{1 / p} \frac{\Gamma\left(\left(\mu+n / p-k+j / p^{\prime}\right) / 2\right)}{\Gamma((\mu+n / p-j / p) / 2)} .
$$

If $p=1$, (3.20) becomes an explicit equality. 
Proof. Let

$$
A=\int_{\mathcal{A}_{n, k}}\left|\left(R_{j, k} f\right)(\tau)\right|^{p}|\tau|^{\nu p} d \tau, \quad B=\int_{\mathcal{A}_{n, j}}|f(\zeta)|^{p}|\zeta|^{\mu p} d \zeta .
$$

By Theorem 2.2,

$$
A^{1 / p} \leq \omega_{j, k, p, \mu}(n) B^{1 / p} .
$$

Our aim is to covert $A$ and $B$ into the corresponding integrals over compact Grassmannians with $R_{j, k} f$ represented by the relevant Funk type transform. We make use of (3.9) with $k$ replaced by $j$ and $\tau$ by $\zeta$. Setting

$$
\varphi(\zeta)=|f(\zeta)|^{p}|\zeta|^{\mu p}, \quad \tilde{h}\left(\zeta_{0}\right) \equiv h\left(\gamma^{-1}\left(\zeta_{0}\right)\right)=\left|\tilde{f}\left(\zeta_{0}\right)\right|^{p}\left(\tan d\left(\zeta_{0}\right)\right)^{\mu p}
$$

we obtain

$$
B=\frac{\sigma_{n}}{\sigma_{j}} \int_{G_{n+1, j+1}} \frac{\left|\tilde{f}\left(\zeta_{0}\right)\right|^{p}\left(\tan d\left(\zeta_{0}\right)\right)^{\mu p}}{\left(\cos d\left(\zeta_{0}\right)\right)^{n+1}} d_{*} \zeta_{0} .
$$

To transform $A$, we observe that by (3.7),

$$
R_{j, k} f=\frac{\sigma_{k}}{\sigma_{j}} \rho_{2} \Lambda_{k}^{-1} F_{j+1, k+1} \Lambda_{j} \rho_{1}^{-1} f
$$

Hence, by (3.7) and (3.9),

$$
\begin{aligned}
A & =\left(\frac{\sigma_{k}}{\sigma_{j}}\right)^{p} \int_{\mathcal{A}_{n, k}}\left|\rho_{2}(\tau)\left(\Lambda_{k}^{-1} F_{j+1, k+1} \Lambda_{j} \rho_{1}^{-1} f\right)(\tau)\right|^{p}|\tau|^{\nu p} d \tau \\
& =\left(\frac{\sigma_{k}}{\sigma_{j}}\right)^{p} \frac{\sigma_{n}}{\sigma_{k}} \int_{G_{n+1, k+1}}\left|\left(F_{j+1, k+1} \Lambda_{j} \rho_{1}^{-1} f\right)\left(\tau_{0}\right)\right|^{p} \\
& \times \frac{\left(\tan d\left(\tau_{0}\right)\right)^{\nu p}\left(\cos d\left(\tau_{0}\right)\right)^{(j+1) p}}{\left(\cos d\left(\tau_{0}\right)\right)^{n+1}} d_{*} \tau_{0}
\end{aligned}
$$

because

$$
\left(\Lambda_{k} \rho_{2}\right)\left(\tau_{0}\right)=\left(1+\left(\tan d\left(\tau_{0}\right)\right)^{2}\right)^{-(j+1) / 2}=\left(\cos d\left(\tau_{0}\right)\right)^{j+1} .
$$

Now we change the notation by setting $\varphi\left(\zeta_{0}\right)=\left(\Lambda_{j} \rho_{1}^{-1} f\right)\left(\zeta_{0}\right)$. Then obvious simplification allows us to write (3.22) in the form

$$
\begin{gathered}
\left(\int_{G_{n+1, k+1}}\left(\sin d\left(\tau_{0}\right)\right)^{\nu p}\left(\cos d\left(\tau_{0}\right)\right)^{(j+1-\nu) p-n-1}\left|\left(F_{j, k} \varphi\right)\left(\tau_{0}\right)\right|^{p} d_{*} \tau_{0}\right)^{1 / p} \\
\leq c\left(\int_{G_{n+1, j+1}}\left(\sin d\left(\zeta_{0}\right)\right)^{\mu p}\left(\cos d\left(\zeta_{0}\right)\right)^{(k+1-\mu) p-n-1}\left|\varphi\left(\zeta_{0}\right)\right|^{p} d_{*} \zeta_{0}\right)^{1 / p} \\
c=\omega_{j, k, p, \mu}(n)\left(\sigma_{j} / \sigma_{k}\right)^{1 / p^{\prime}}
\end{gathered}
$$


To complete the proof, it remains to replace $n+1$ by $n, k+1$ by $k$, and $j+1$ by $j$. The equality sign in (3.20), when $p=1$, holds by comment 3 in Subsection 2.1.

Let us set $\varphi=F_{j} \psi$ in (3.20), where $\psi$ is a function on $S^{n-1}$. Using the equality $F_{j, k} F_{j} \psi=F_{k} \psi$, we obtain the following result.

Corollary 3.7. Let $j, k, n, \alpha, \beta$ and $c$ be the same as in Theorem 3.6. Then

$$
\left(\int_{G_{n, k}}\left|\left(F_{k} \psi\right)\left(\tau_{0}\right)\right|^{p} \alpha\left(\tau_{0}\right) d_{*} \tau_{0}\right)^{1 / p} \leq c\left(\int_{G_{n, j}}\left|\left(F_{j} \psi\right)\left(\zeta_{0}\right)\right|^{p} \beta\left(\zeta_{0}\right) d_{*} \zeta_{0}\right)^{1 / p}
$$

provided that the integral on the right-hand side exists in the Lebesgue sense.

For further purposes, we formulate a particular case of Theorem 3.6 corresponding to $j=1$, when a function $\varphi$ on $G_{n, 1}$ can be identified with an even function on $S^{n-1}$ and the evenness restriction can be dropped, as we did in (3.19).

Theorem 3.8. Let $0<k<n, \tau_{0} \in G_{n, k}, \theta=\left(\theta_{1}, \ldots, \theta_{n}\right) \in S^{n-1}$. We set

$$
\begin{gathered}
\alpha_{1}\left(\tau_{0}\right)=\left(\sin d\left(\tau_{0}\right)\right)^{\nu p}\left(\cos d\left(\tau_{0}\right)\right)^{(1-\nu) p-n} \\
\beta_{1}(\theta)=\left(1-\theta_{n}^{2}\right)^{\mu p / 2}\left|\theta_{n}\right|^{(k-\mu) p-n}
\end{gathered}
$$

and suppose that

$$
1 \leq p \leq \infty, \quad \nu=\mu-(k-1) / p^{\prime}, \quad \mu>k-n / p-1 / p^{\prime} .
$$

Then for every measurable function $\varphi$ on $S^{n-1}$,

$$
\left(\int_{G_{n, k}}\left|\left(F_{k} \varphi\right)\left(\tau_{0}\right)\right|^{p} \alpha_{1}\left(\tau_{0}\right) d_{*} \tau_{0}\right)^{1 / p} \leq c_{1}\left(\int_{S^{n-1}}|\varphi(\theta)|^{p} \beta_{1}(\theta) d_{*} \theta\right)^{1 / p}
$$

where $c_{1}$ is a sharp constant having the form

$$
c_{1}=\left(\frac{\Gamma(k / 2)}{\Gamma(1 / 2)}\right)^{1 / p^{\prime}}\left(\frac{\Gamma((n-1) / 2)}{\Gamma((n-k) / 2)}\right)^{1 / p} \frac{\Gamma\left(\left(\mu+n / p-k+1 / p^{\prime}\right) / 2\right)}{\Gamma((\mu+n / p-1 / p) / 2)} .
$$

If $p=1$, (3.26) becomes an explicit equality. Specifically,

$$
\int_{G_{n, k}}\left(F_{k} \varphi\right)\left(\tau_{0}\right) \tilde{\alpha}_{1}\left(\tau_{0}\right) d_{*} \tau_{0}=\tilde{c}_{1} \int_{S^{n-1}} \varphi(\theta) \tilde{\beta}_{1}(\theta) d_{*} \theta
$$

where

$\tilde{\alpha}_{1}\left(\tau_{0}\right)=\left(\sin d\left(\tau_{0}\right)\right)^{\mu}\left(\cos d\left(\tau_{0}\right)\right)^{1-\mu-n}, \quad \tilde{\beta}_{1}(\theta)=\left(1-\theta_{n}^{2}\right)^{\mu / 2}\left|\theta_{n}\right|^{k-\mu-n}$, 


$$
\tilde{c}_{1}=\frac{\Gamma((n-1) / 2)}{\Gamma((n-k) / 2)} \frac{\Gamma((\mu+n-k) / 2)}{\Gamma((\mu+n-1) / 2)}, \quad \mu>k-n
$$

Choosing $\mu, \nu, p, k$ and $n$ in Theorem 3.8 in a suitable way, one can obtain a series of new inequalities with sharp constants.

Example 3.9. Let $\nu=0, p=n$. Then (3.26) yields

$$
\left(\int_{G_{n, k}}\left|\left(F_{k} \varphi\right)\left(\tau_{0}\right)\right|^{n} d_{*} \tau_{0}\right)^{1 / n} \leq c_{0,1}\left(\int_{S^{n-1}}|\varphi(\theta)|^{n} \beta_{0}(\theta) d_{*} \theta\right)^{1 / n},
$$

where

$$
\begin{gathered}
\beta_{0}(\theta)=\left(1-\theta_{n}^{2}\right)^{(k-1)(n-1) / 2}\left|\theta_{n}\right|^{k-1} \\
c_{0,1}=\left(\frac{\Gamma(k / 2)}{\Gamma(1 / 2)}\right)^{1-1 / n}\left(\frac{\Gamma((n-1) / 2)}{\Gamma((n-k) / 2)}\right)^{1 / n} \frac{\Gamma((1-k / n) / 2)}{\Gamma((k-k / n) / 2)} .
\end{gathered}
$$

Another result can be obtained if we apply Theorem 3.6 to functions of the form $\varphi=F_{j} \psi$ which are Funk transforms over $(j-1)$-dimensional geodesics in $S^{n-1}$. Noting that $F_{j, k} \varphi=F_{j, k} F_{j} \psi=F_{k} \psi$, we obtain the following inequality connecting Funk transforms over geodesics of different dimensions.

Theorem 3.10. Suppose that $j, k, \mu, \nu, p, \alpha, \beta$ and $c$ have the same meaning as in Theorem 3.6. Then

$$
\left(\int_{G_{n, k}}\left|\left(F_{k} \psi\right)\left(\tau_{0}\right)\right|^{p} \alpha\left(\tau_{0}\right) d_{*} \tau_{0}\right)^{1 / p} \leq c\left(\int_{G_{n, j}}\left|\left(F_{j} \psi\right)\left(\zeta_{0}\right)\right|^{p} \beta\left(\zeta_{0}\right) d_{*} \zeta_{0}\right)^{1 / p} .
$$

\section{Cross-Sections of Star Sets}

4.1. Preliminaries. Theorems of the previous section imply a host of geometric inequalities and equalities. Below we give some examples. But first we need to establish terminology and recall some known facts.

A subset $L$ of $\mathbb{R}^{n}$ is called a star set (with respect to the origin) if $\lambda x \in L$ for every $x \in L$ and every $\lambda \in[0,1]$. A star set $L$ is uniquely determined by its radial function

$$
\rho_{L}(\theta)=\sup \{c \geq 0: c \theta \in L\}, \quad \theta \in S^{n-1} .
$$

Everywhere in the following, we assume the set $L$ to be good enough so that the Lebesgue integrals on the right-hand side of our formulas are finite. It means that $\rho_{L}$ belongs to the corresponding Lebesgue space on the sphere. For example, Klain [38, Definition 2.3] considered the so-called $L^{p}$-stars for which $\rho_{L} \in L^{p}\left(S^{n-1}\right)$. 
For every star set $L \subset \mathbb{R}^{n}$, the volume (i.e., the Lebesgue measure) of $L$ can be expressed in polar coordinates as

$$
V_{n}(L)=\frac{1}{n} \int_{S^{n-1}} \rho_{L}^{n}(\theta) d \theta=b_{n} \int_{S^{n-1}} \rho_{L}^{n}(\theta) d_{*} \theta .
$$

Similarly, for $0<k<n$ and $\tau_{0} \in G_{n, k}$, the volume of the central cross-section $L \cap \tau_{0}$ is

$$
V_{k}\left(L \cap \tau_{0}\right)=b_{k} \int_{S^{n-1} \cap \tau_{0}} \rho_{L}^{k}(\theta) d_{\tau_{0}} \theta=b_{k}\left(F_{k} \rho_{L}^{k}\right)\left(\tau_{0}\right),
$$

where $F_{k}$ is the Funk transform (3.18). The corresponding $p$-means

$$
\left(\int_{G_{n, k}}\left[V_{k}\left(L \cap \tau_{0}\right)\right]^{p} d_{*} \tau_{0}\right)^{1 / p}
$$

were introduced by Lutwak [40, 41] and have proved to be useful in various geometrical considerations; see, e.g., [13], [26, Section 9.4 and Note 9.7 on p. 384]. Natural generalizations of (4.1) and (4.2) are $m$ th dual elementary mixed volumes (or $m$ th dual Quermassintegrals)

$$
\begin{gathered}
\tilde{V}_{m}(L)=b_{n} \int_{S^{n-1}} \rho_{L}^{m}(\theta) d_{*} \theta=\frac{m}{n} \int_{L}|x|^{m-n} d x, \\
\tilde{V}_{m}\left(L \cap \tau_{0}\right)=b_{k} \int_{S^{n-1} \cap \tau_{0}} \rho_{L}^{m}(\theta) d_{\tau_{0}} \theta=b_{k}\left(F_{k} \rho_{L}^{m}\right)\left(\tau_{0}\right), \quad \tau_{0} \in G_{n, k},
\end{gathered}
$$

which were introduced by Lutwak $[39,42]$ and studied by many authors; see, e.g., [6, p. 158], [26, p. 409] and references therein. In particular, (4.4)-(4.5) naturally arise in the study of the Busemann-Petty type comparison problems for convex bodies [35, 59]. The quantity (4.4) can also be treated as the $m$-homogeneous rotation invariant valuation $[1,38]$. Clearly,

$$
\tilde{V}_{n}(L)=V_{n}(L) ; \quad \tilde{V}_{k}\left(L \cap \tau_{0}\right)=V_{k}\left(L \cap \tau_{0}\right) .
$$

By the well-known property of the Funk transform [52],

$$
\int_{G_{n, k}}\left(F_{k} f\right)\left(\tau_{0}\right) d_{*} \tau_{0}=\int_{S^{n-1}} f(\theta) d_{*} \theta,
$$

(4.4) and (4.2) yield

$$
\tilde{V}_{k}(L)=\frac{b_{n}}{b_{k}} \int_{G_{n, k}} V_{k}\left(L \cap \tau_{0}\right) d_{*} \tau_{0}
$$


(set $f=\rho_{L}^{k}$ in (4.7) and make use of (4.4) and (4.5)).

4.2. Weighted Estimates. We start with the following

Remark 4.1. All inequalities in this subsection hold with sharp constants. The sharpness is guaranteed by comment $\mathbf{1}$ in Subsection 2.1 because every nonnegative function on the sphere can be regarded as a radial function of some star set. One should note that if we impose additional restrictions on the class of star sets, rather than finiteness of the corresponding integrals, the sharpness of the constants becomes unknown. For example, we do not know if our constants are sharp in the class of star bodies, when $\rho_{L}$ is continuous.

If $L$ is a star set, we can apply Theorem 3.8 to functionals (4.1)-(4.5). For example, setting $\varphi=\rho_{L}^{m}$ in (3.26) and using (4.5), we obtain

$$
\int_{G_{n, k}}\left[\tilde{V}_{m}\left(L \cap \tau_{0}\right)\right]^{p} \alpha_{1}\left(\tau_{0}\right) d_{*} \tau_{0} \leq\left(c_{1} b_{k}\right)^{p} \int_{S^{n-1}} \rho_{L}^{m p}(\theta) \beta_{1}(\theta) d_{*} \theta,
$$

where $\alpha_{1}, \beta_{1}, k, p, \tau_{0}$ and $c_{1}$ are the same as in (3.26). If $p=1,(4.9)$ becomes an explicit equality

$$
\int_{G_{n, k}} \tilde{V}_{m}\left(L \cap \tau_{0}\right) \tilde{\alpha}_{1}\left(\tau_{0}\right) d_{*} \tau_{0}=\tilde{c}_{1} b_{k} \int_{S^{n-1}} \rho_{L}^{m}(\theta) \tilde{\beta}_{1}(\theta) d_{*} \theta,
$$

where $\tilde{\alpha}_{1}, \tilde{\beta}_{1}$ and $\tilde{c}_{1}$ have the same meaning as in (3.28). For the sake of convenience, we recall

$$
\begin{gathered}
\tilde{\alpha}_{1}\left(\tau_{0}\right)=\left(\sin d\left(\tau_{0}\right)\right)^{\mu}\left(\cos d\left(\tau_{0}\right)\right)^{1-\mu-n}, \quad \tilde{\beta}_{1}(\theta)=\left(1-\theta_{n}^{2}\right)^{\mu / 2}\left|\theta_{n}\right|^{k-\mu-n}, \\
\tilde{c}_{1}=\frac{\Gamma((n-1) / 2)}{\Gamma((n-k) / 2)} \frac{\Gamma((\mu+n-k) / 2)}{\Gamma((\mu+n-1) / 2)}, \quad \mu>k-n .
\end{gathered}
$$

If $\mu=0,(4.10)$ becomes

$$
\int_{G_{n, k}} \tilde{V}_{m}\left(L \cap \tau_{0}\right) \frac{d_{*} \tau_{0}}{\left(\cos d\left(\tau_{0}\right)\right)^{n-1}}=b_{k} \int_{S^{n-1}} \rho_{L}^{m}(\theta) \frac{d_{*} \theta}{\left|\theta_{n}\right|^{n-k}} .
$$

In particular, if $m=k,(4.6)$ yields

$$
\int_{G_{n, k}} V_{k}\left(L \cap \tau_{0}\right) \frac{d_{*} \tau_{0}}{\left(\cos d\left(\tau_{0}\right)\right)^{n-1}}=b_{k} \int_{S^{n-1}} \rho_{L}^{k}(\theta) \frac{d_{*} \theta}{\left|\theta_{n}\right|^{n-k}} .
$$

Further, choosing $p=n / k$ and $\mu=0$ in (4.9), we obtain

$$
\int_{G_{n, k}}\left[\tilde{V}_{m}\left(L \cap \tau_{0}\right)\right]^{n / k} \alpha_{2}\left(\tau_{0}\right) d_{*} \tau_{0} \leq \frac{\left(c_{2} b_{k}\right)^{n / k}}{b_{n}} \tilde{V}_{m n / k}(L),
$$


where

$$
\begin{gathered}
\alpha_{2}\left(\tau_{0}\right)=\left(\sin d\left(\tau_{0}\right)\right)^{(k-1)(k-n) / k}\left(\cos d\left(\tau_{0}\right)\right)^{1-k} \\
c_{2}=\left(\frac{\Gamma(k / 2)}{\Gamma(1 / 2)}\right)^{1-k / n}\left(\frac{\Gamma((n-1) / 2)}{\sigma_{n-1} \Gamma((n-k) / 2)}\right)^{k / n} \frac{\Gamma((1-k / n) / 2)}{k \Gamma((k-k / n) / 2)} .
\end{gathered}
$$

If $m=k$, (4.9) becomes

$$
\int_{G_{n, k}}\left[V_{k}\left(L \cap \tau_{0}\right)\right]^{p} \alpha_{1}\left(\tau_{0}\right) d_{*} \tau_{0} \leq\left(c_{1} b_{k}\right)^{p} \int_{S^{n-1}} \rho_{L}^{k p}(\theta) \beta_{1}(\theta) d_{*} \theta,
$$

where all parameters have the same meaning as in (3.26). In particular, for $p=n / k$ and $\mu=0$,

$$
\int_{G_{n, k}}\left[V_{k}\left(L \cap \tau_{0}\right)\right]^{n / k} \alpha_{2}\left(\tau_{0}\right) d_{*} \tau_{0} \leq c_{2}^{n / k} b_{k}^{n / k-1} \operatorname{vol}_{n}(L),
$$

where $\alpha_{2}$ and $c_{2}$ are the same as in (4.13).

We conclude this subsection by exhibiting a nice inequality for central sections of different dimensions. This inequality follows from (3.25), (4.2), and (4.5), if we set $\psi=\rho_{L}^{j}$. Specifically,

$$
\int_{G_{n, k}}\left[\tilde{V}_{j}\left(L \cap \tau_{0}\right)\right]^{p} \alpha\left(\tau_{0}\right) d_{*} \tau_{0} \leq\left(\frac{c b_{k}}{b_{j}}\right)^{p} \int_{G_{n, j}}\left[V_{j}\left(L \cap \zeta_{0}\right)\right]^{p} \beta\left(\zeta_{0}\right) d_{*} \zeta_{0},
$$

where all parameters have the same meaning as in Theorem 3.6. An interested reader may derive many consequences of (4.16) by choosing different combinations of parameters.

4.3. Unweighted Estimates. Let us write (3.19) for $\varphi=\rho_{L}^{k}$, where $L$ is a measurable star set in $\mathbb{R}^{n}$ of finite measure. We obtain

$$
\left\|F_{k} \rho_{L}^{k}\right\|_{n} \leq\left\|\rho_{L}^{k}\right\|_{n / k}
$$

or, by (4.1) and (4.2),

$$
\int_{G_{n, k}}\left[V_{k}\left(L \cap \tau_{0}\right)\right]^{n} d_{*} \tau_{0} \leq \frac{b_{k}^{n}}{b_{n}^{k}}\left[V_{n}(L)\right]^{k} .
$$

The inequality (4.18) has an interesting history. If $k=n-1$ and $L$ is a convex body, it was proved by Busemann [7], and is known as the Busemann intersection inequality. The case of convex bodies with any $0<k<n$ is due to Busemann and Straus [8] and Grinberg [30]; see also Gardner [26, Corollary 9.4.5]. The equality sign in (4.18) yields the celebrated Furstenberg-Tzkoni formula [25, 46] for ellipsoids. Further 
progress was made in Gardner's work [27], where (4.18) was extended to arbitrary bounded Borel subsets of $\mathbb{R}^{n}$.

Our approach to (4.18), that relies on the corresponding inequality for the $k$-plane transform, shows that (4.18) actually holds for arbitrary (not necessarily bounded) star set $L$ of finite measure.

For applications of (4.18), the reader is referred to $[10,13,15,26$, $27,50,51,70]$. A similar inequality on the sphere and the hyperbolic spaces was studied by Dann, Kim and Yaskin [14].

Following Lutwak's observation [43, p. 162, (3)], one can proceed in the opposite direction that may give an alternative proof of the DruryChrist-Drouot inequality (2.17). Suppose that (4.18) has been proved "geometrically" for star bodies $L$ with smooth boundary. Then, given a smooth nonnegative function $\varphi$ on $S^{n-1}$, we can define a star set $L$ with the radial function $\rho_{L}=\varphi^{1 / k}$ and get $\left\|F_{k} \varphi\right\|_{n} \leq\|\varphi\|_{n / k}$. The density argument extends this estimate to all $\varphi \in L^{n / k}\left(S^{n-1}\right)$, and the stereographic projection in Theorem 3.3 yields $(2.17)$.

Further, for all $1 \leq j<k<n$, setting $\varphi\left(\zeta_{0}\right)=\left(F_{j} \rho_{L}^{k}\right)\left(\zeta_{0}\right)=$ $b_{j}^{-1} \tilde{V}_{k}\left(L \cap \zeta_{0}\right)$ in (3.17) and using the equality

$$
\left(F_{j, k} F_{j} \rho_{L}^{k}\right)\left(\tau_{0}\right)=\left(F_{k} \rho_{L}^{k}\right)\left(\tau_{0}\right)=b_{k}^{-1} V_{k}\left(L \cap \tau_{0}\right),
$$

we arrive at the following conjecture generalizing (4.18).

Conjecture 4.2. For any measurable star set $L$ in $\mathbb{R}^{n}$ and any $1 \leq$ $j<k<n$,

$$
\int_{G_{n, k}}\left[V_{k}\left(L \cap \tau_{0}\right)\right]^{n / j} d_{*} \tau_{0} \leq\left(\frac{b_{k}}{b_{j}}\right)^{n / j}\left(\int_{G_{n, j}}\left[\tilde{V}_{k}\left(L \cap \zeta_{0}\right)\right]^{n / k} d_{*} \zeta_{0}\right)^{k / j} .
$$

More generally, choosing $\varphi\left(\zeta_{0}\right)=\left(F_{j} \rho_{L}^{m}\right)\left(\zeta_{0}\right)=b_{j}^{-1} \tilde{V}_{m}\left(L \cap \zeta_{0}\right)$, we have

$$
\int_{G_{n, k}}\left[\tilde{V}_{m}\left(L \cap \tau_{0}\right)\right]^{n / j} d_{*} \tau_{0} \leq\left(\frac{b_{k}}{b_{j}}\right)^{n / j}\left(\int_{G_{n, j}}\left[\tilde{V}_{m}\left(L \cap \zeta_{0}\right)\right]^{n / k} d_{*} \zeta_{0}\right)^{k / j} .
$$

Here $m$ is an arbitrary real number for which the integral on the righthand side exists in the Lebesgue sense.

If (4.19) and (4.20) are true, they are sharp because $\varphi \equiv 1$ yields the equality sign in (3.17).

Acknowledgement. I am thankful to Artem Zvavitch who brought my attention to the preprint [14]. 


\section{REFERENCES}

1. S. Alesker, Continuous rotation invariant valuations on convex sets. Annals of Math. 149(1999), 977-1005.

2. A. Baernstein, II and M. Loss, Some conjectures about $L^{p}$ norms of $k$-plane transforms. Rend. Sem. Mat. Fis. Milano 67(1997), 9-26.

3. J. Bennett, N. Bez and S. Gutirrez, Transversal multilinear Radon-like transforms: local and global estimates. Rev. Mat. Iberoam. 29(2013), 765-788.

4. C. A. Berenstein, E. Casadio Tarabusi and A. Kurusa, Radon transform on spaces of constant curvature. Proc. Amer. Math. Soc. 125(1997), 455-461.

5. G. Bianchi, R. Gardner and P. Gronchi, Symmetrization in geometry. Adv. Math. 306(2017), 51-88.

6. Yu. D. Burago and V. A. Zalgaller, Geometric Inequalities, Springer (2014).

7. H. Busemann, Volume in terms of concurrent cross-sections. Pacific J. Math. 3(1953), 1-12.

8. H. Busemann and E. G. Straus, Area and normality. Pacific J. Math. 10(1960), $35-72$.

9. A. P. Calderón, On the Radon transform and some of its generalizations. In Conference on harmonic analysis in honor of Antoni Zygmund, Vol. I, II (Chicago, Ill., 1981), Wadsworth Math. Ser. (Wadsworth, Belmont, CA, 1983), $673-689$.

10. G. Chasapis, A. Giannopoulos and D.- M. Liakopoulos, Estimates for measures of lower dimensional sections of convex bodies. Adv. Math. 306(2017), 880-904.

11. M. Christ, Estimates for the k-plane transform. Indiana Univ. Math. J. 33(1984), 891-910.

12. M. Christ, Extremizers of a Radon transform inequality. In Advances in Analysis: The Legacy of Elias M. Stein, Princeton Math. Ser., 50 (Princeton Univ. Press, Princeton, NJ, 2014), 87-109.

13. N. Dafnis and G. Paouris, Estimates for the affine and dual affine quermassintegrals of convex bodies. Illinois J. Math. 56(2012), 1005-1021.

14. S. Dann, J. Kim and V. Yaskin, Busemann's intersection inequality in hyperbolic and spherical spaces. Preprint 2017, arXiv:1706.06776.

15. S. Dann, G. Paouris and P. Pivovarov, Bounding marginal densities via affine isoperimetry. Proc. Lond. Math. Soc. 113(3)(2016), 140-162.

16. A. Drouot, Sharp constant for a k-plane transform inequality. Anal. PDE 7(2014), 1237-1252.

17. A. Drouot, Quantitative form of certain k-plane transform inequalities. $J$. Funct. Anal. 268(2015), 1241-1276.

18. A. Drouot, Existence and non-existence of extremizers for certain $k$-plane transform inequalities, Preprint, 2016, arXiv:1412.4876v2.

19. S. W. Drury, $L^{p}$ estimates for the $X$-ray transform. Illinois J. Math. 27(1983), $125-129$.

20. S. W. Drury, Generalizations of Riesz potentials and Lp estimates for certain k-plane transforms. Illinois J. Math. 28(1984), 495-512.

21. S. W. Drury, An endpoint estimate for certain k-plane transforms. Canad. Math. Bull. 29(1986), 96-101.

22. S. W. Drury, A survey of $k$-plane transform estimates. In Commutative harmonic analysis, Contemp. Math. 91, NY: Canton, (1987) (Amer. Math. Soc., Providence, RI 1989), 43-55. 
23. S. W. Drury, $L^{p}$ estimates for certain generalizations of $k$-plane transforms. Illinois J. Math. 33(3)(1989), 367-374.

24. T.C. Flock, Uniqueness of extremizers for an endpoint inequality of the k-plane transform. J. Geom. Anal. 26(2016), 570-602.

25. H. Furstenberg and I. Tzkoni, Spherical functions and integral geometry. Israel J. Math. 10(1971), 327-338.

26. R. J. Gardner, Geometric Tomography, 2nd edn., Cambridge University Press (New York, 2006).

27. R. J. Gardner, The dual Brunn-Minkowski theory for bounded Borel sets: dual affine quermassintegrals and inequalities. Adv. Math. 216(2007), 358-386.

28. F. B. Gonzalez, Notes on Integral geometry and Harmonic Analysis. COE Lecture Note, 24. Math-for-Industry Lecture Note Series. Kyushu University, Faculty of Mathematics (Fukuoka, 2010).

29. F. B. Gonzalez and T. Kakehi, Pfaffian systems and Radon transforms on affine Grassmann manifolds. Math. Ann. 326(2)(2003), 237-273.

30. M.I. Graev, A problem of integral geometry related to a triple of Grassmann manifolds. Functional Analysis and its Applications, 34(4)(2000), 299-301.

31. P.T. Gressman, Sharp $L^{p}-L^{q}$ estimates for generalized $k$-plane transforms. $A d v$. Math. 214(2007), 344-365.

32. P.T. Gressman, Uniform sublevel Radon-like inequalities. J. Geom. Anal. 23(2013), 611-652.

33. I. S. Gradshteyn and I. M. Ryzhik. Table of Integrals, Series and Products (Academic Press, 1980).

34. E. L. Grinberg, Isoperimetric inequalities and identities for k-dimensional crosssections of convex bodies. Math. Ann. 291(1991), 75-86.

35. H. Hadwiger, Radialpotenzintegrale zentralsymmetrischer Rotations-körper und Ungleichheitsaussagen Busemannscher Art. (German). Math. Scand. 23(1968), 193-200.

36. S. Helgason, Integral geometry and Radon transform, Springer, (New YorkDordrecht-Heidelberg-London, 2011).

37. F. Keinert. Inversion of $k$-plane transforms and applications in computer tomography. SIAM Review 31(1989), 273-289.

38. D.A. Klain, Star valuations and dual mixed volumes. Advances in Math. 121(1996), 80-101.

39. E. Lutwak, Dual mixed volumes. Pacific J. Math. 58(1975), 531-538.

40. E. Lutwak, Mean dual and harmonic cross-sectional measures. Ann. Mat. Pura Appl. 119(4)(1979), 139-148.

41. E. Lutwak, Inequalities for Hadwiger's harmonic Quermassintegrals. Math. Ann. 280(1988), 165-175.

42. E. Lutwak, Intersection bodies and dual mixed volumes. Adv. in Math. 71(1988), 232-261.

43. E. Lutwak, Selected affine isoperimetric inequalities. In Handbook of convex geometry, Vol. A, B, North-Holland (Amsterdam, 1993) 151-176.

44. A. Markoe, Analytic Tomography. Encyclopedia of Mathematics and its Applications 106, Cambridge Univ. Press (New York, 2006).

45. S.G. Mikhlin, Multidimensional singular integrals and integral equations, Fizmatgiz (Moscow, 1962). 
46. R. E. Miles, A simple derivation of a formula of Furstenberg and Tzkoni. Israel J. Math. 14(1973), 278-280.

47. D. M. Oberlin, $L^{p}-L^{q}$ mapping properties of the Radon transform. In Banach spaces, harmonic analysis, and probability theory (Storrs, Conn., 1980/1981), Lecture Notes in Math., 995, Springer, (Berlin, 1983), 95-102.

48. D. M. Oberlin and E. M. Stein, Mapping properties of the Radon transform. Indiana Univ. Math. J. 31(1982), 641-650.

49. R. Oberlin, Two bounds for the X-ray transform. Math. Z. 266(2010), 623-644.

50. G. Paouris and P. Pivovarov, Randomized isoperimetric inequalities. Preprint 2016, arXiv:1607.00519 [math.MG].

51. G. Paouris and P. Valettas, Neighborhoods on the Grassmannian of marginals with bounded isotropic constant. J. Funct. Anal. 267(2014), 3427-3443.

52. B. Rubin, Inversion formulas for the spherical Radon transform and the generalized cosine transform. Advances in Appl. Math. 29(2002), 471-497.

53 . B. Rubin, Reconstruction of functions from their integrals over $k$-dimensional planes. Israel J. of Math. 141(2004), 93-117.

54. B. Rubin, Radon transforms on affine Grassmannians. Trans. Amer. Math. Soc. 356(2004), 5045-5070.

55. B. Rubin, Weighted norm inequalities for k-plane transforms. Proc. Amer. Math. Soc. 142(2014), 3455-3467.

56. B. Rubin, Introduction to Radon transforms (with elements of fractional calculus and harmonic analysis), Cambridge University Press (New York, 2015).

57. B. Rubin and Y. Wang, On Radon transforms between lines and hyperplanes. Intern. Journal of Math. (to appear), arXiv:1601.03826v3 [math.FA].

58. B. Rubin and Y. Wang, New inversion formulas for Radon transforms on affine Grassmannians. Preprint 2016, arXiv:1610.02109 [math.FA].

59. B. Rubin and Gaoyong Zhang, Generalizations of the Busemann-Petty problem for sections of convex bodies. J. Funct. Anal. 213(2)(2004), 473-501.

60. R. Schneider, Inequalities for random flats meeting a convex body. J. Appl. Probab. 22(1985), 710-716.

61. R. Schneider, Convex Bodies: The Brunn-Minkowski Theory, 2nd edn, Cambridge Univ. Press (New York, 2013).

62. R. Schneider and W. Weil, Stochastic and Integral Geometry, Springer (BerlinHeidelberg, 2008).

63. D. C. Solmon, The $X$-ray transform. J. Math. Anal. Appl. 56(1976), 61-83.

64. D. C. Solmon, A note on $k$-plane integral transforms. Journal of Math. Anal. and Appl. 71(1979), 351-358.

65. E. M. Stein, Singular integrals and differentiability properties of functions, Princeton Univ. Press (Princeton, NJ, 1970).

66. R.S. Strichartz, $L^{p}$-estimates for Radon transforms in Euclidean and noneuclidean spaces. Duke Math. J. 48(1981), 699-727.

67. R.S. Strichartz, Harmonic analysis on Grassmannian bundles. Trans. of the Amer. Math. Soc. 296(1986), 387-409.

68. T. Tao and J. Wright, $L^{p}$ improving bounds for averages along curves. J. Amer. Math. Soc. 16(2003), 605-638.

69. F. G. Tricomi and A. Erdélyi, The asymptotic expansion of a ratio of gamma functions. Pacific J. Math. 1(1951), 133-142. 
70. G. Zhang, Geometric inequalities and inclusion measures of convex bodies. Mathematika 41(1994), 95-116.

Department of Mathematics, Louisiana State University, Baton Rouge, LA, 70803 USA

E-mail address: borisr@math.lsu.edu 